\title{
Estudio de los procesos de creación léxica en el ámbito oleoturístico (francés-español)
}

\author{
Francisco Luque Janodet \\ Universidad de Sevilla \\ fco.luque.janodet@gmail.com \\ https://dx.doi.org.10.12795/futhark.2018.il 3.05
}

Fecha de recepción: 10.05.2018

Fecha de aceptación: 21.07 .2018

Resumen: El turismo es uno de los sectores más importantes de la economía española. Su diversidad ha propiciado la creación de rutas temáticas que potencian el turismo de interior y rural usando como eje central un determinado producto, como el aceite de oliva o el vino. Esto ha dado lugar a nuevos conceptos y realidades que las lenguas tratan de denominar a través de diferentes recursos léxicos. El presente artículo estudia la creación de neologismos en el ámbito del oleoturismo desde una perspectiva sincrónica a partir de un corpus textual comparable y bilingüe ad hoc y de un posterior extracto de términos. En esta contribución, tras haber presentado los dieciocho neologismos más destacables, se analizan los procesos de creación léxica más habituales en este sector turístico. Este estudio concluye que el procedimiento más usual en la creación de términos especializados en el par de lenguas de lenguas francés-español es la composición culta.

Palabras clave: creación léxica, oleoturismo, neologismo.

\section{Study of the lexical creation processes in oleotourism sector (French-Spanish)}

Abstract: Tourism is one of the most important sectors in the Spanish economy. The diversity of the tourist sector has led to the creation of thematic routes that enhance inland and rural tourism using as a central axis a specific product, such as olive oil or wine. This has given rise to new concepts and realities that languages try to name through different lexical resources. This paper studies the creation of neologisms in the field of Oleotourism from a synchronic perspective and from a comparable and bilingual corpus. Thus, the most common lexical creation 
processes in this tourism sector are analyzed. This study concludes that the most usual procedure in the creation of specialized terms in the French-Spanish languages is the word composition.

Key words: lexical creation, oleotourism, neologism.

Sumario: I. Introducción. 2. El sector turístico en España. 3. Aceite de oliva: recurso económico y patrimonio cultural. 4. Consideraciones en torno a la naturaleza del neologismo y a los procedimientos de creación léxica. 5. Objetivos y metodología. 6. Análisis y resultados. 7. Conclusiones.

\section{Introducción}

A lo largo de los últimos años, el sector turístico en España ha vivido un verdadero auge que le ha permitido erigirse como una de las principales actividades económicas del país. Parte de su éxito se debe, además, a su versatilidad y amplitud. El turismo de sol y playa ya no es el mayor reclamo del sector, puesto que encontramos una oferta diversificada que incluye el turismo rural, el turismo temático, el turismo de interior o el gastronómico. De esta manera, las distintas regiones de interior se sirven de su patrimonio histórico o natural para atraer a turistas nacionales e internacionales a partir de la creación de rutas que conjugan el arte y la arquitectura civil y religiosa, así como sus productos artesanales y locales, como el vino y el aceite de oliva. De hecho, las distintas bodegas y almazaras, regiones y denominaciones de origen han sabido aprovechar este gran potencial turístico, lo cual ha dado lugar al enoturismo y al oleoturismo. En el caso del primero, se han creado distintas rutas enoturísticas, como, por ejemplo, la Ruta del Vino de las Rías Baixas, de Jumillo, de Somontano, la Ruta del vino Ribera del Duero o la Ruta del Vino de Montilla-Moriles, entre otras. Por otra parte, en el ámbito del oleoturismo, actividad que centrará el presente artículo, se han creado las Vías Verdes en Jaén, la Ruta del aceite de oliva de Córdoba o la Ruta del aceite de la Sierra (Cádiz). Dichos itinerarios dan a conocer al visitante los productos típicos de la región, ofrecen actividades variadas y potencian el turismo de interior. Asimismo, la creación de distintas actividades o de espacios temáticos ha propiciado la creación de nuevos vocablos, neologismos, a partir de los diferentes medios de los que disponen las lenguas francesa y española, los cuales nos proponemos analizar desde un punto de vista sincrónico. Debemos destacar que se trata de una línea de investigación que parte de los estudios previos de Sabater Abad (2016), quien abordó la creación léxica en el ámbito enoturístico y su inclusión en obras lexicográficas y en manuales de español para fines específicos. 


\section{El sector turístico en España}

El turismo se ha postulado como una de las principales actividades económicas en España. El potencial turístico de este país queda reflejado en las cifras aportadas por el Instituto Nacional de Estadística (2017), según las cuales España recibió 75,3 millones de turistas internacionales en 2016, con un aumento del $10,3 \%$ respecto al año anterior. Asimismo, de acuerdo con el portavoz del Gobierno de España y Ministro de Educación, Cultura y Deporte, Íñigo Méndez de Vigo, España podría alcanzar la cifra de 83 millones de turistas en caso de mantenerse el ritmo de crecimiento de los dos primeros meses de 2017. El turismo es, por tanto, una actividad en auge, dinámica y versátil en nuestro país.

Asimismo, a partir de los datos presentados por SAETA, la Empresa Pública de Turismo y Deporte de Andalucía, y de la Encuesta de Coyuntura Turística de Andalucía, se extrae que la comunidad autónoma registró un total de 17,8 millones de viajeros en establecimientos hoteleros. Las expectativas de este sector son, además, halagüeñas, ya que España recibió, en 2017, un total de 81,8 millones de turistas extranjeros, con un aumento del $8,6 \%$ con respecto al año anterior.

\section{Aceite de oliva: recurso económico y patrimonio cultural}

España es, de acuerdo con el Ministerio de Agricultura y Pesca, Alimentación y Medio Ambiente (MAGRAMA), el primer exportador mundial de aceite de oliva. Las exportaciones totales, que incluyen a más de 100 países, representan el $60 \%$ del comercio total de este producto. Asimismo, de acuerdo con este Ministerio, la balanza comercial es positiva, con un saldo de I 743 millones de euros en 2012 y un volumen de exportaciones que ha aumentado un $48 \%$ desde la campaña 2006/2007 hasta la campaña 201 I/2012.

No debemos olvidar que nos encontramos ante un producto de gran tradición en España $y$, particularmente en Andalucía, como han puesto de manifiesto Nieto, Garrido, Padial y Carrilero (1995), quienes documentaron que parte de las construcciones estudiadas en la villa romana de Las Vías (Cuevas del Becerro, Málaga) tenían como objetivo la obtención de aceite de oliva. La tradición de este producto ahonda sus raíces en toda la cuenca mediterránea, pues como señala Bolens (1996: 181):

El aceite de unción de los hebreos, de Jesucristo, de los reyes francos y de las suras coránicas era un aceite de oliva milenario. En el alfabeto hebreo, el buey es Aleph, la casa Beith, el camello Guimel, y con el número 7 como valor aparece el aceite, representado por el Zaïn: el aceite está implicado en esta letra de los primeros alfabetos semíticos, entre los polos de la civilización. 
Transmite la diea de vibración, de oro, de esplendor, el caldeo alegría y belleza, y zaït en hebreo (la misma palabra en árabe): olivo, aceituna y aceite, es decir, la esencia luminosa.

Su comercio es también histórico en Europa, tal como afirma esta autora:

El comercio de aceite de oliva es el prototipo del movimiento comercial: los escitas de la estepa meridional rusa acudían a abastecerse al mediterráneo oriental; los almacenes de ánforas, como los de Komo en Crita, confirman la importancia del comercio. Vid y olivo son sinónimos de civilización (Bolens, 1996: 181).

Además, su historia y arraigo local en las distintas regiones productoras ha comenzado a recogerse en distintos catálogos y bases de datos. Ello puede comprobarse mediante la consulta del Atlas de Patrimonio Inmaterial de Andalucía, una base de datos del patrimonio cultural e inmaterial dependiente del Instituto Andaluz del Patrimonio Histórico de la Junta de Andalucía. Se trata de una fuente de información abierta del patrimonio de los distintos municipios y provincias de la Comunidad Autónoma en el que hay documentados un total de I 752 bienes en cuatro grandes ámbitos o categorías temáticas: rituales festivos, oficios y saberes, modos de expresión y, por último, alimentación y sistemas culinarios. Su consulta permite corroborar la protección de la producción de aceite en Martos (Jaén), en Morón de la Frontera y en Osuna (Sevilla), en Andévalo (Huelva), en Antequera (Málaga), o en Filabres-Tabernas (Almería), así como la recogida de la aceituna en Pedrera (Sevilla) dentro de la categoría de Oficios y saberes.

Por tanto, su tradición sociocultural e histórica en España $y$, particularmente, en Andalucía, así como el incremento de los movimientos de turistas nacionales e internacionales han permitido la creación de distintas rutas gastronómicas que Barrera (2006 apud Millán y Agudo, 2010: 96) clasifica en:

I. Rutas gastronómicas por productos, es decir, rutas organizadas basadas en un producto determinado.

2. Rutas gastronómicas de playo, basadas en un plato preparado.

3. Rutas étnico-gastronómicas, cuyo sustento es la tradición culinaria de pueblos emigrantes.

De esta manera, como indican Millán y Agudo (2010: 97), los entes públicos han desarrollado una serie de programas con el fin de facilitar las perspectivas turísticas en las zonas olivareras, como Oleoadapt, Oleoyouthstart, Oleointegra, Oleointerpresise y Oleolife; asimismo, ponen de manifiesto que desde la Diputación de Jaén se lidera un proyecto basado en la creación de una red de intercambio de experiencias en el que participan Portugal, Grecia, Francia, Croacia, Túnez, Marruecos y España. Por tanto, el aceite de oliva y el olivar se han visto 
afectadas por el auge del turismo rural (Millán y Agudo, 2010: 97) gracias al cual se puede entrar en "viejas almazaras convertidas en lujosos restaurantes y hoteles rurales, bodegas y patios de labranza convertidos en museos y salas de degustación. El turismo del aceite se presenta como una nueva faceta emergente [...]" (2010: 97-98). A pesar de ello, los estudios de Millán, Morales y Agudo (2010: 22) han concluido que el número de almazaras abiertas al público sigue siendo reducido $y$, por tanto, la oferta del oleoturismo no satisface la demanda actual, pese a que el perfil del turista es de un visitante fiel y con una demanda estacional. No obstante, es fundamental que destacar que esta actividad puede ser "una oportunidad de desarrollo de actividades económicas complementarias [...]" (Millán et al., ibid.), que sirva para potenciar el turismo de interior y rural de las numerosas denominaciones de origen españolas.

\section{Consideraciones en torno a la naturaleza del neologismo y a los procedimientos de creación léxica}

Los discursos oleícola y olivarero son la manifestación lingüística de una comunidad epistemológica especializada formada por los distintos oficios del aceite $y$ del olivar, entre ellos, olivicultores, vareadores, catadores, etc. Nos encontramos, por tanto, ante un discurso histórico y con arraigo local, pero altamente productivo, pues se adapta a las nuevas realidades de este sector a partir de la formación de neologismos. En esta línea, coincidimos con Křečková (1997) cuando afirma que «les progrès scientifique, technique et culturel de notre siècle ont eu pour effet la création d'un nombre important de termes nouveaux». En este sentido, como indica esta autora, un neologismo aparece cuando un nace un nuevo concepto tras el descubrimiento de un investigador. De esta manera, este concepto se denomina de forma consciente por el descubridor, comienza a circular en el medio científico y requiere de una denominación en idiomas extranjeros; aunque podríamos ampliar dicha definición a aquellas realidades surgidas no necesariamente en el ámbito científico y académico, como es, por ejemplo, el oleoturismo.

En lo que respecta a la caracterización del neologismo y de la neología, Azorín y Sánchez (2016: 16) definen estos conceptos como:

El proceso mediante el cual se crea un recurso lingüístico nuevo, no existente con anterioridad en la lengua, recibe el nombre de neología; mientras que el recurso creado, que puede ser léxico, sintáctico, fónico, etc., se conoce como neologismo.

No obstante, de acuerdo con Cabré (1993), la consideración de una palabra o término como neologismo solo tiene lugar en el seno de la Terminología si existen cuatro condiciones previas: 
I) que haya aparecido recientemente (diacronía);

2) que sea percibida como una unidad nueva por los hablantes (psicología);

3) que no aparezca en los diccionarios (lexicografía);

4) que presente inestabilidad morfológica, gráfica, fonética y/o semántica.

Asimismo, Cabré (2006) contempla distintas categorías para su clasificación:

a) Neologismos de forma (sufijación, prefijación, interferencias entre sufijación y prefijación, composición, composición culta, lexicalización, conversión sintáctica, sintagmación, siglación, acronimia, abreviación, variación).

b) Neologismos sintácticos, que implican un cambio de subcategoría gramática en una base léxica;

c) Neologismos semánticos, es decir, aquellos formados por una modificación del significado de una base léxica, o aquellos formados a partir de un nombre propio usado como nombre común;

d) Préstamo y préstamo adaptado;

e) Otros, esto es, las palabras simples, dialectales, argóticas, cultismos - casos difíciles de etiquetar pero que, en su esencia, son neológicas; si bien es cierto que, como señala Křečková (1997), la formación de términos se realiza siguiendo los mismos mecanismos de formación de palabras de la lengua general).

Asimismo, existen para esta autora (ibid.) tres procesos de formación de palabras que pueden dar lugar a neologismos: I) derivación, 2) composición y 3) abreviación. No obstante, según Cabré (1993), debemos distinguir entre neologismos, entendidos como creaciones léxicas propias de la lengua general, y los neónimos, términos creados por especialistas en un ámbito de especialidad con unidades polilexemáticas y de formación culta, necesarios para denominar un nuevo producto o realidad, que responden a una necesidad denominativa, de uso duradero y que rechazan la sinonimia. Rondeau (1984) señala, a su vez, dos tipos de neónimos: I) los neónimos de origen (néonymes d'origine), creados por especialistas de un ámbito, y 2) los neónimos de apoyo (néonymes d'appoint), adaptados a otro código lingüístico $o$ a una nueva situación conceptual y describe tres tipos de formación neonímica: I) modos de formación morfológica (derivación y apócope), 2) modos de formación morfosintáctica (agrupamiento sintagmático, cambio de categoría gramatical, reducción) y 3 ) modos de formación morfosemántica (calco, préstamo). 


\section{Objetivos y metodología}

El presente proyecto pretende estudiar los neologismos surgidos en el ámbito del oleoturismo, entendido como una actividad turística centrada en el aceite oliva y en el olivar, a partir del análisis de los procedimientos de creación léxica. Para la consecución del presente proyecto, hemos partido de los estudios de Sabater Abad (2016) y de la metodología empleada por esta autora. Por tanto, hemos compilado un corpus ad hoc neológico, comparable y bilingüe (francésespañol), obtenido fundamentalmente de textos descargados de internet siguiendo la metodología protocolizada de Seghiri Domínguez (20II). La selección y extracción de términos se ha realizado desde el punto de vista intuitivo. Asimismo, los neologismos han sido cribados mediante la consulta en la versión en línea el Diccionario de la lengua española de la Real Academia Española.

\section{Análisis y resultados}

Habiendo analizado el corpus comparable neológico, hemos podido extraer un total de 22 neologismos en español y II términos en francés. La importancia del sector olivarero en España frente a dicho sector en Francia puede ayudar a explicar el desequilibrio cuantitativo del extracto de términos. Asimismo, debemos considerar que, pese a que existen países francófonos en la cuenca mediterránea que dedican parte de su sector primario a la producción de aceite de oliva, su confluencia con el turismo sigue encontrándose en fase de desarrollo.

Consideradas estas cuestiones y descrito el corpus de trabajo, procederemos a analizar los términos más relevantes que han sido extraídos, considerando la categoría gramatical y el mecanismo de formación implicado:

\section{Almazara museo}

Categoría gramatical: sustantivo.

Mecanismo de formación: se trata de un neologismo creado por composición. Está formado por los sustantivos almazara y museo.

\section{Ecoturismo}

Categoría gramatical: sustantivo.

Mecanismo de formación: formación por composición culta mediante el prefijo griego eco- (de ốkos, 'casa') y el sustantivo turismo. 
3. Molino museo

Categoría gramatical: sustantivo.

Mecanismo de formación: neologismo creado por composición, formado por los sustantivos molino y museo.

\section{Oleopaquete}

Categoría gramatical: sustantivo.

Mecanismo de formación: neologismo creado por composición culta, mediante el prefijo oleo- (del latín oleum) y paquete.

\section{Oleospa}

Categoría gramatical: sustantivo.

Mecanismo de formación: neologismo formado por composición culta, mediante el prefijo oleo- (del latín oleum) y spa.

\section{Oleotaberna}

Categoría gramatical: sustantivo.

Mecanismo de formación: neologismo creado por composición culta mediante el prefijo oleo- (del latín oleum) y taberna.

\section{Oleocultura}

Categoría gramatical: sustantivo.

Mecanismo de formación: neologismo formado por composición culta, mediante el prefijo oleo- (del latín oleum) y taberna.

\section{Oleoescuela}

Categoría gramatical: sustantivo.

Mecanismo de formación: neologismo creado por composición culta, mediante el prefijo oleo- (del latín oleum) y escuela. 
9. Oleo ruta

Categoría gramatical: sustantivo.

Mecanismo de formación: neologismo creado por sintagmación, a partir del prefijo culto oleo- (del latín oleum) y ruta.

Variantes: oleorruta.

\section{Oleoteca}

Categoría gramatical: sustantivo.

Mecanismo de formación: neologismo creado por composición culta, mediante el prefijo oleo- (del latín oleum) y la raíz-teca (del griego Өńkn, thêke 'armario'.

Variantes: olivoteca.

\section{Oleoterapia}

Categoría gramatical: sustantivo.

Mecanismo de formación: neologismo creado por composición culta, mediante el prefijo oleo- (del latín oleum) y el sustantivo terapia.

Variantes: olivaterapia.

\section{Oleoturismo}

Categoría gramatical: sustantivo.

Mecanismo de formación: neologismo creado por composición culta, mediante el prefijo oleo- (del latín oleum) y el sustantivo turismo

\section{Ruta del olivo}

Grupo sintáctico: sintagma nominal.

Mecanismo de formación: neologismo creado por sintagmación (composición), mediante el sustantivo ruta unido mediante el artículo contracto del y el sustantivo olivo. Sigue una combinación nombre + nombre. 
14. Vía Verde del Aceite

Grupo sintáctico: sintagma nominal.

Mecanismo de formación: neologismo creado por sintagmación (composición), mediante el sustantivo vía y el adjetivo verde unidos mediante el artículo contracto del al sustantivo aceite. Sigue una combinación nombre + nombre.

Una vez analizados los términos en español, realizaremos un estudio de cuatro ejemplos extraídos del corpus francés:

15. Musée du moulin à huile

Grupo sintáctico: sintagma nominal.

Mecanismo de formación: neologismo creado por sintagmación (composición), mediante el sustantivo musée ('museo') unido mediante el artículo contracto du al sustantivo moulin à huile ('almazara', 'molino de aceite'). Dicho término se ha formado, a su vez, por un procedimiento similar mediante la unión de los sustantivos moulin ('molino') y huile ('aceite') mediante la preposición à. Sigue una combinación nombre + nombre.

\section{Oléothérapie}

Categoría gramatical: sustantivo.

Mecanismo de formación: neologismo creado por composición culta, mediante el prefijo oléo- (del latín oleum) y el sustantivo thérapie.

\section{Oléotourisme}

Categoría gramatical: sustantivo.

Mecanismo de formación: neologismo creado por composición culta, mediante el prefijo oléo- (del latín oleum) y el sustantivo tourisme.

18. Tourisme oléicole

Categoría gramatical: sustantivo. 
Mecanismo de formación: neologismo creado sintagmación, siguiendo el modelo nombre + adjetivo, es decir, el sustantivo tourime y el adjetivo oléicole. Dicho adjetivo se ha formado, a su vez, por composición culta, mediante el prefijo oléo(del latín oleum) y la raíz -cole.

\section{Conclusiones}

El aceite de oliva es un producto que ha acompañado a las civilizaciones de la cuenca mediterránea a lo largo de milenios. No obstante, es un producto que el turismo "ha redescubierto" y se ha servido de él para potenciar el turismo de interior en detrimento del tradicional turismo de sol y playa. Se trata, pues, de un recurso del que pueden hacer uso regiones como Córdoba, Jaén o Sevilla para atraer a turistas nacionales e internacionales, mediante la combinación con el ecoturismo, la creación de rutas temáticas o la realización de catas de aceite.

A la luz de los resultados obtenidos, podemos concluir que la composición culta es el procedimiento de creación léxica más productivo en el ámbito del oleoturismo tanto en español como en francés. No obstante, debemos señalar que los procesos de composición destacan por una fuerte inestabilidad, puesto que no existe una estandarización de los neologismos hallados en el conjunto de textos que forman el corpus. El empleo del prefijo oleo- se puede deber, como ha señalado Sabater (2016: 85), a una cuestión de prestigio, ya que "estas nuevas perspectivas que se le quieren dar llevan adheridas una especie de hedonismo revestido de cierto renombre y elegancia". Finalmente, hemos constatado una diferencia cuantitativa significante en el par de idiomas de trabajo, fundamentalmente porque nos encontramos ante un sector más potenciado en España que en los países francófonos.

\section{Referencias bibliográficas}

Azorín, D. (2003): "Neologismos incorporados por Salvá en el Nuevo Diccionario de la lengua castellana". ELUA, 17: 107-139.

Barrera, E. (2006) "Turismo Rural: nueva ruralidad y empleo rural no agrícola". Montevideo, Cinterfor/OIT.

Bolens, L. (1996): "Riquezas de la tierra andaluza y primacía del aceite de oliva en la sociedad y la civilización de al-Andalus (siglos X-XVI)". Agricultura y Sociedad 80-8I: $|8|-2 \mid 6$.

Cabré, M. T. (2006): "La clasificación de neologismos: una tarea compleja". Alfa, 50(2): 229-250. (1993): La terminología: teoría, metodología, aplicaciones. Barcelona: Antártida Empúries. 
Consejo de Ministros (07/04/2017): "El Gobierno regula la concesión de subvenciones para la asistencia jurídica gratuita". Disponible en http://www.lamoncloa.gob.es/consejodeministros/resumenes/Pagina.

Empresa pública para la gestión del turismo y del deporte de Andalucía (2017): Total turismo en Andalucía. Datos básicos del turismo en Andalucía. Junta de Andalucía, Consejería de Turismo y Deporte. Disponible en: http://www.juntadeandalucia.es/turismoydeporte/publicaciones/estadisticas/ perfil_prof_total_turistas_jun I7.pdf.

Instituto Nacional de Estadística (2017): "España recibe 4,0 millones de turistas internacionales en diciembre, un 13,3\% más que en el mismo mes de 2015". Disponible Web: htup:/mwwinees/daco/daco42/fronturlfronturl216.pdf

Junta de Andalucía (s. f.): Catálogo del Patrimonio Inmaterial de Andalucía. Instituto Andaluz del Patrimonio Histórico, Consejería de Cultura. Disponible en: http://www.iaph.es/patrimonio-inmaterial-andalucia/frmSimple.do.

Millán, M. ${ }^{a}$ G., Agudo E. M. (2010). "El turismo gastronómico y las Denominaciones de origen en el sur de España: Oleoturismo. Un estudio de caso". Pasos: Revista de Turismo y Patrimonio Cultural 8: 9I-I I2.

Millán, M.a G., Fernández, E., Agudo E. V. (2010). "El oleoturismo como motor de desarrollo rural. La denominación de origen de Montoro-Adamuz". Mundo Agrario, 2I, vol. II: I-27.

Ministerio de Agricultura, Pesca y Alimentación (2017): "Aceite de oliva". Disponible en: hups//wnw.mapagob.es/es/agicultura/temas/producciones-agicolas/aceiteolva-y-aceituna-mesa/aceiteaspx

Nieto, B., Garrido O., Padial, B., Carrilero, M. (1995). "La villa romana de Las Viñas (Cuevas del Becerro, Málaga) y el poblamiento romano en la depresión de Ronda". Florentia iliberritana: Revista de estudios de antigüedad clásica, 6: 89. 108.

Real Academia Española (2017): Diccionario de la lengua española (23. ${ }^{a}$ edición) [en línea]. Disponible en: <dle.rae.es>

Rondeau, G. (1984): Introduction à la terminologie, Paris, Gaëtan Morin.

Sabater, C. (2016). La creación léxica en el ámbito del enoturismo y su inclusión en obras lexicográficas y en manuales de español para fines específicos. Trabajo Fin de Máster. Alicante: Universidad de Alicante.

Seghiri, M. (20II). "Metodología protocolizada de compilación de un corpus de seguros de viajes: aspectos de diseño y representatividad". RLA: Revista de Lingüística Teórica y Aplicada 49 (2): 13-30.

Křečková, V. (1997): "Les tendances de la néologie terminologique en français contemporain". Studia minora facultatis philosophicae Universitatis Brunensis, L. 18: 63-69. 